\title{
Conflits de proximité et rapport(s) au(x) droit(s)
}

Proximity conflicts and their relations to right(s)

\section{Antonio Azuela, Patrice Melé et Vicente Ugalde}

\section{(2) OpenEdition}

Journals

Édition électronique

URL : http://journals.openedition.org/developpementdurable/10787

DOI : 10.4000/developpementdurable.10787

ISSN : 1772-9971

Éditeur

Association DD\&T

\section{Référence électronique}

Antonio Azuela, Patrice Melé et Vicente Ugalde, «Conflits de proximité et rapport(s) au(x) droit(s) », Développement durable et territoires [En ligne], Vol.6, n¹ | Mars 2015, mis en ligne le 31 mars 2015, consulté le 02 mai 2019. URL : http://journals.openedition.org/developpementdurable/10787 ; DOI : 10.4000/developpementdurable.10787

Ce document a été généré automatiquement le 2 mai 2019.

Développement Durable et Territoires est mis à disposition selon les termes de la licence Creative Commons Attribution - Pas d'Utilisation Commerciale 4.0 International. 


\title{
Conflits de proximité et rapport(s) $\mathrm{au}(\mathrm{x}) \operatorname{droit}(\mathrm{s})$
}

\author{
Proximity conflicts and their relations to right(s) \\ Antonio Azuela, Patrice Melé et Vicente Ugalde
}

1 Plusieurs recherches récentes menées par les auteurs de cet article ont porté sur les effets de situations dans lesquelles des collectifs composés d'habitants, de militants, de représentants politiques locaux se mobilisent pour contrôler le devenir de certains espaces, pour s'opposer à des projets ou pour demander la protection de sous-ensembles spatiaux. Si nous avons choisi de regrouper ces situations sous le terme générique de conflits de proximité, il ne s'agit pas de considérer le «proche » comme susceptible d'une délimitation territoriale ou d'une mesure objective par le chercheur. La définition de la proximité que nous retenons est celle donnée par les résidents ou usagers lorsqu'ils affirment qu'un projet, une activité, une pratique affecte leur environnement ${ }^{1}$. Cela implique que la distance entre les objets, activités ou pratiques mises en cause peut être plus ou moins grande (voisinage non désiré, mobilisation pour la préservation d'un paysage, bruit des avions, pollution atmosphérique ou des nappes phréatiques...). Néanmoins, tous les conflits considérés ici se manifestent par des actions collectives durant lesquelles des résidents effectuent un travail de (re)définition de leur inscription spatiale et territoriale.

Dans le cadre de programmes de recherche collectifs, nous avons choisi d'analyser dans une perspective internationale (Canada, France, Italie, Mexique) ces situations à partir d'une problématique centrée sur l'analyse de leurs effets et des relations entre conflits, participation et décisions. L'objectif de cet article est de rendre compte d'une façon synthétique des résultats de ces recherches en ce qui concerne les effets des situations de conflits, d'une part, sur les rapports au droit des populations et des institutions, et, d'autre part, sur la place des invocations de droits par les groupes mobilisés. Les hypothèses d'interprétation formulées dans cet article sur la productivité juridique des situations de conflits reposent donc sur l'analyse d'études de cas sur différents types de conflits ${ }^{2}$. Néanmoins, la place impartie ici ne nous permettra pas d'inclure des références précises aux situations étudiées, nous renvoyons pour cela le lecteur aux travaux publiés 
(Melé, 2011 ; 2013) où en cours de publication sur cette thématique (Bobbio, Melé, Ugalde, 2015). Il ne sera pas non plus possible de développer ici des références précises aux différents contextes juridiques, ni aux spécificités des acteurs du monde du droit et de la construction du droit de l'environnement dans différents contextes nationaux (Azuela, 2006).

3 Pour une réflexion sur les relations entre droit et géographie, l'analyse de situations de conflits de proximité semble particulièrement pertinente à plusieurs niveaux. En effet, ce sont des situations dans lesquelles des groupes de résidents tentent de construire des prises sur le devenir de certains espaces, revendiquent le maintien d'ordres locaux face à l'incertitude ou à la redistribution des nuisances. Par ailleurs, les procédures administratives et juridiques sont mises à l'épreuve à partir de leur capacité à protéger les habitants des nuisances et des pollutions, à rendre possible ou à empêcher certaines dynamiques urbaines. De plus, les différentes modalités de régulations des usages du sol et de l'attribution des autorisations de localisation des activités sont au cœur des débats. Dans un contexte de généralisation des conflits malgré le renforcement du cadre juridique environnemental et la prégnance du référentiel de la participation, l'enjeu de cette réflexion est donc d'analyser la place du droit dans les recompositions des processus de localisation et de territorialisation dans les sociétés contemporaines.

Nous proposons donc d'intégrer aux débats sur les dimensions juridiques des relations à l'espace et sur les dimensions spatiales et territoriales du droit, nos hypothèses d'interprétation sur la place du droit dans les situations de conflits locaux construites à partir de la confrontation entre nos études de cas et différentes formes de problématisation de ses phénomènes au sein des sciences sociales.

\section{Apparaître dans l'espace public et politique}

5 Le premier constat que l'on doit faire est celui de la multiplicité des formes de présences, d'usages et de mobilisations du droit. Dans certains cas, des procédures sont engagées pour faire intervenir un tribunal ; dans d'autres cas, il s'agit d'une utilisation plus diffuse du droit, dans les médias, dans des réunions publiques, sur internet, afin de publiciser une prise de position, d'ancrer une revendication.

6 Il est, en effet, possible de considérer la présence du droit dans des situations d'action collective en dehors de tout recours contentieux. Les situations de conflit et mobilisation sont des moments d'exposition d'habitants ordinaires à l'ordre juridique, épreuves qui sont aussi des moments de socialisation juridique. Pour les habitants, le droit existe d'abord comme texte dont ils attendent un effet dans leur confrontation avec les représentants des pouvoirs publics. Plusieurs situations décrites par des acteurs interrogés, montrent que non seulement ils construisent une interprétation du droit directement à partir du texte de loi, mais qu'ils ont éprouvé en situation la force du droit comme texte et leur capacité à le mobiliser. Produire dans un débat ou une controverse un texte - ayant une plus ou moins forte valeur juridique - pour tenter de délégitimer une pratique administrative ou d'ancrer une revendication constitue l'une des expériences les plus communes de cette fonction du droit. L'expérience du droit est alors celle d'un corpus dans lequel il est possible de puiser pour fonder ses droits. Il s'agit donc ici de faire exister localement le cadre juridique, plus exactement les textes de lois considérés littéralement comme affirmation explicite. Apparaît ici l'un des usages sociaux du droit : une des caractéristiques du droit est en effet d'être un texte disponible soumis à 
interprétation directe par la lecture. À ce niveau de l'analyse, les textes de lois existent en eux-mêmes, indépendamment des attributs qui peuvent les transformer en acte juridique ou en politique publique (décrets d'applications, règlements, procédures et pratiques administratives). On peut penser que cette exposition au droit comme texte a des effets sur la rationalité des habitants mobilisés, sur leur vision du monde, sur la définition des situations. En filigrane, c'est la dimension cognitive du droit qui se dégage, c'est-à-dire le droit conçu comme un langage qui permet de rendre intelligible une réalité, qui fournit un espace de dialogue entre des intérêts divergents et structure l'intervention des pouvoirs publics.

7 L'utilisation du droit comme forum qui permet de publiciser des postures et de les légitimer, c'est-à-dire de traduire en termes de droit des revendications qui sans cela seraient moins légitimes, est un enjeu observable dans presque tous les cas étudiés dans nos travaux. Cette forme de recours au droit donne également lieu à une variété de situations, mais on peut identifier deux objectifs principaux : d'une part, l'idée de « faire pression" sur le gouvernement en publicisant l'affaire ou en contestant des actes d'exercice du pouvoir (autorisations, non respect des procédures); d'autre part, la construction d'une légitimité en évoquant l'attribution de droits subjectifs dont seraient porteurs les intentions des législateurs et la distance entre cette perception de l'attribution de droits et l'application concrète de la législation.

8 L'invocation du droit semble être considérée comme un instrument efficace, indépendamment de son effectivité. L'usage symbolique du droit ne se réduit pas aux représentations qui circulent dans l'espace public, mais ce que nous rappellent les études de cas c'est aussi que l'efficacité symbolique du droit dépend de son usage tactique. La peur de la stigmatisation en public fait agir les acteurs, et de cette façon la dimension juridique contribue à l'organisation du conflit, même lorsque les tribunaux compétents n'interviennent pas pour dire qui doit faire quoi. Néanmoins, certaines conditions sont requises pour que ce recours au droit s'avère efficace. On ne peut pas dire que la nécessité légale ait partout la même forme et la même intensité. Dans certains pays, les acteurs sont moins enclins à agir par peur d'être stigmatisés; la place des références à une opinion publique active, devant laquelle il serait possible de dénoncer des décisions ou des comportements des pouvoirs publics, est aussi différente suivant les contextes nationaux.

9 Par ailleurs, la construction de la légitimité des acteurs (dans ce cas, surtout des opposants à un projet) passe aussi par le recours à des institutions autres que les tribunaux (plaintes plus ou moins formelles, recours administratifs, demande d'information auprès de commissions). Cette stratégie tout azimut permet de rester présent dans le conflit et d'appuyer son argumentation sur les avis d'organismes officiels. Par le recours à des figures qui relèvent du droit à l'information, du droit de l'homme ou de l'application du droit de l'environnement et de l'urbanisme, les acteurs cherchent également à renforcer leurs positions dans un espace publicisé de confrontation.

\section{Les effets de la judiciarisation}

10 Pour les groupes mobilisés, le contact avec le monde du droit et ses représentants dans le cadre de recours juridiques est une expérience ambivalente. Considérons aussi qu'un conflit se transforme lorsqu'un différend est judiciarisé. On peut d'abord évoquer des effets de cadrage, des processus d'apprentissage du langage du droit mais aussi 
d'élaboration collective d'une situation juridique, par l'intermédiaire d'interactions avec des professionnels du droit qui statuent sur les arguments qu'il est possible de traduire en stratégies juridiques. Cette expérience transforme la place du droit dans la construction d'un cadre cognitif; les tribunaux, en effet, ont vocation à proposer une seule interprétation ou qualification légitime d'une situation, rompant ainsi la continuité entre les représentations juridiques et le reste des représentations qui prévalaient avant qu'un cas leur soit soumis. Par ailleurs, entrant en contact avec les acteurs du champ juridique, les groupes mobilisés font l'expérience de l'importance des procédures, qui souvent semblent prévaloir sur les arguments de justice sociale, spatiale ou environnementale qu'ils tentent de faire reconnaître.

11 Ce que produit le "passage du droit ", c'est bien comme le note Bruno Latour (2002) en conclusion de son ethnographie du Conseil d'État, de rattacher une situation ou un conflit particuliers à la totalité des précédents et à l'ensemble du corpus du droit administratif ; processus qui nécessite des reformulations, des traductions, des redéfinitions d'une situation particulière.

On peut ensuite envisager les effets à caractère « distributif » que certains faits juridiques peuvent avoir sur le développement des conflits. Il s'agit ici de la fonction d'arbitrage du droit, processus assez classique mais finalement peu fréquent,. Les décisions adoptées par les juges et d'autres actes strictement juridiques peuvent avoir des effets directs sur un conflit. Pour les juristes et les opérateurs du droit, il s'agit là du fonctionnement normal de l'ordre juridique. Dans le langage et les représentations du champ juridique, le droit sert précisément à "résoudre » des conflits. Si un promoteur et un groupe de riverains s'affrontent autour d'un projet d'infrastructure, il existe la possibilité qu'un juge ou un organe administratif adopte une décision qui remette "les choses à leur place " qui désigne des gagnants et des perdants, qui puisse donc clore la controverse en distribuant des biens et des peines. Pour utiliser une définition ancienne de la justice, la décision peut " donner à chacun ce qui lui revient ».

13 Il s'agit là de faits juridiques qui peuvent être considérés comme distributifs, au sens ou ils orientent le conflit à partir de ce qu'ils donnent ou enlèvent aux différents acteurs. Il importe pour nous de souligner l'importance de ce niveau, car il faut rappeler qu'un certain regard sociologique a tendance à minimiser ces faits. C'est le cas en particulier de la sociologie des mouvements sociaux qui considère que les mobilisations sociales et non des phénomènes d'ordre juridique produisent les effets déterminants. Comme Charles Tilly et d'autres auteurs l'ont signalé, cette sociologie s'est surtout focalisée sur les aspects transgressifs des mouvements sociaux, en laissant de côté l'étude de la façon dont les acteurs mobilisés utilisaient en leur faveur les ressources que leur offrait l'ordre juridique (McAdam, Tarrow, Tilly, 2001).

14 Il est vrai que certains arguments peuvent conduire à relativiser l'importance des faits juridiques. D'abord, il faut signaler que les conflits qui sont « résolus » par les procédures prévues par les lois sont en fait peu nombreux. En effet, pour cela, plusieurs conditions doivent être réunies. Les acteurs doivent avoir connaissance de ces dispositifs et accès à des avocats capables de les activer efficacement; les juges ou leurs équivalents doivent être disposés à les reconnaître, et surtout il faut que des procédures de recours soient disponibles, c'est-à-dire, qu'elles existent au sein de l'ordre juridique positif considéré.

Même dans le champ de la sociologie juridique, il existe une certaine résistance à reconnaitre l'importance des effets distributifs de certains faits juridiques. C'est le cas, 
par exemple des travaux qui en cherchant à comprendre les nouvelles fonctions sociales du droit et en mobilisant la théorie des systèmes ont proclamé la fin de la fonction régulatrice $\mathrm{du}$ droit $^{3}$. Il est néanmoins certain que les conditions pour qu'existe un fonctionnement - classique selon les juristes - de l'ordre juridique comme source de solution à des conflits, sont relativement rares et c'est pour cela que de nombreux conflits prennent place en dehors de l'ordre juridique.

16 Malgré leur force ces arguments ne sont pas suffisants pour diminuer l'importance des cas dans lesquels certains faits juridiques marquent le développement des conflits de proximité. En d'autres termes, même s'il s'agit de cas peu fréquents, il existe des situations dans lesquelles le droit est objet d'actualisation dans un conflit de proximité, au sens ici d'activation des procédures de recours offrant un canal aux conflits. Et il est intéressant d'observer ces cas, non seulement, pour comprendre les conditions qui les ont rendu possibles, mais aussi, parce que la confiance dans la capacité du droit à remplir cette fonction reste forte, bien au-delà du monde des juristes.

\section{Utilisation des indéterminations du droit}

17 Un autre argument qui justifierait un scepticisme sociologique face à l'optimisme ou à l'ingénuité des juristes - en ce qui concerne la capacité du droit à trancher les conflits est lié à l'indétermination qui caractérise l'expérience juridique. Il n'est ni possible de savoir ce que sera le résultat de la décision juridique ni ce que sera son effet. Une solution qui peut paraitre définitive dans les termes du droit peut dans la pratique avoir un impact limité sur les relations sociales. Il est aussi possible qu'une décision qui soit présentée comme provisoire devienne définitive si elle reste en vigueur suffisamment longtemps ${ }^{4}$. En fait, le traitement juridique d'un conflit peut être un élément aussi contingent que ses autres dimensions. Ainsi, par exemple, ce qui peut apparaître comme une "solution " dans le monde du droit, n'en est pas nécessairement une. Une sentence, au lieu de résoudre un conflit peut l'aggraver, le rendre plus aigu. Ce type de situations, finalement assez fréquent, alimente une critique du droit tout en reconnaissant implicitement, paradoxalement, son efficacité.

En fait, l'indétermination du droit est une des hypothèses les plus largement acceptés par les études du droit qui considère le droit comme un langage porteur d'imprécision et dont le sens fluctue. Cette indétermination ne relève ni des lacunes ni de l'exercice discrétionnaire du droit mais plutôt de l'attribution d'un sens au terme utilisé dans le texte juridique, et se révèle notamment dans les cas-limites. La question de l'indétermination du droit est au cœur, depuis de nombreuses décennies, des travaux de doctrine juridique ${ }^{5}$. Le mérite d'auteurs tels que Kelsen (1962) ou Hart (2006) est de mieux saisir cette notion et d'en comprendre les implications. Au risque de simplifier des analyses complexes, nous en retiendrons deux points clés: d'abord, l'idée que le droit, loin d'être un ensemble de textes statiques prêts à être appliqués, est au contraire dynamique; les changements du droit (sous l'effet du législateur ou encore des interprétations du juge entre autres) participent à une indétermination tant dans l'application de la règle que dans sa définition. Ensuite, la "texture ouverte $»^{6}$ (open texture) du droit est en soi à l'origine de nombreuses indéterminations: la nature du langage juridique laisse une marge de manœuvre aux juges pour interpréter la règle ce qui rend difficile l'anticipation des effets juridiques d'une action ou de connaître le résultat d'une action judiciaire. 
Nous ne nous attarderons pas sur les sources d'indétermination juridique, c'est-à-dire sur les caractéristiques propres au droit qui font que l'on ne peut espérer du droit qu'il fonctionne comme un ensemble cohérent de dispositifs, mais nous pouvons souligner qu'une grande partie des controverses juridiques reposent sur des tentatives d'utilisation par les acteurs de cette caractéristique du droit. En profitant des interstices laissés par l'indétermination du droit, certains acteurs, par leurs usages au droit, participent à son indétermination.

Par ailleurs, nous observons dans de nombreux cas, des tentatives pour modifier les règles. C'est le cas en particulier lorsque la position de certains acteurs mobilisés change et leur donne accès à des postes où ils exercent une certaine autorité. Dans l'ensemble des contextes étudiés les conflits de proximité constituent des formes de politisation et des représentants des opposants peuvent se retrouver en position de gestion politique au niveau local ou construire des alliances avec des acteurs politiques. L'arrivée d'une nouvelle personnalité politique à un poste exécutif peut changer les conditions juridiques d'un projet, mais aussi les stratégies pour modifier les règles et en particulier celles liées aux documents de planification territoriale ou à la place accordée à des dispositifs de participation ou de concertation.

21 Même si dans certains cas, il s'agit de règles secondaires d'application (Lascoumes, 1990), ces exemples mettent en évidence que l'immutabilité du droit est plus le produit de la représentation de certains acteurs qu'une propriété des systèmes juridiques, puisque les autorités, tout en respectant le cadre juridique, peuvent modifier les règles qui s'appliquent à des espaces et, par conséquent, les conditions sous lesquelles le droit sert de cadre à la formulation des attentes des acteurs. Le changement de règles se présente ainsi comme une action sur le cadre juridique mais qui peut être perçu comme créant un flou et une instabilité juridique. Le fait de revenir sur des autorisations ou sur les règles plus générales instaure un système où il est difficile pour les acteurs de prévoir les réactions et la direction des projets.

Si l'on se place du point de vue d'un entrepreneur qui demande une autorisation pour son activité, il peut considérer que les conditions d'une stabilité juridique ne sont pas totalement assurées et se retourner vers la justice, y compris par des canaux supranationaux. Pour les opposants à un projet, rien non plus ne certifie qu'une fois arrêté le projet ne reprenne pas à un moment ou un autre. Cette instabilité et ce flou juridiques obligent les acteurs à réadapter et à diversifier constamment leurs stratégies de recours au droit.

Par leurs stratégies diversifiées, les acteurs participent à leur tour à l'indétermination du droit. Nous en avons repéré deux principales dans l'ensemble des cas, l'une qui a des impacts sur les temporalités d'un conflit, et l'autre, plus directement liée à l'indétermination du droit, concernant les luttes pour définir ou redéfinir des concepts restés flous. Dans des contextes très différents et sur des objets très diversifiés, nous avons pu voir que les recours en justice peuvent durer longtemps. Très souvent, il ne s'agit pas pour les parties en jeu d'obtenir un résultat concret dans un sens ou l'autre qui motive mais plutôt de rallonger le processus de prise de décision. La même stratégie est utilisée dans l'espace administratif. Il ne s'agit pas tant de faire accepter sa position que de la rendre publique et de gagner du temps. Gagner du temps, mais pourquoi faire ? La réponse est aussi multiple que les motivations en présence. Il nous semble que, toujours dans l'optique d'observer les rapports au droit, il s'agit parfois de ralentir le projet en espérant la mise en place de nouvelles règlementations. 

fond. C'est le cas des dispositifs de participation sociale, d'accès à l'information, de consultation publique, et surtout, de la tendance à faciliter l'accès à la justice par la reconnaissance des intérêts diffus ${ }^{8}$. Ces nouvelles procédures ont été instaurées précisément dans l'objectif de canaliser les conflits et d'éviter les débordements, et souvent, elles sont proposées comme réponses aux demandes des organisations sociales que se mobilisent sur les différentes thématiques qui produisent des conflits de proximité.

\section{Actualisation locale du droit}

Dans plusieurs situations étudiées, le cadre de régulation des activités - commissions consultatives, zonages inscrits dans les plans, prescriptions liées à des espaces protégés, différentes procédures de médiation ou d'information du public - est présenté par les habitants comme le résultat d'une mobilisation pour une meilleure application des règles existantes et pour une meilleure prise en compte de leurs droits. Les groupes impliqués 
affichent comme objectif la construction d'un ordre local qui permette de contraindre les modalités de localisation de nouveaux usages, mais aussi le fonctionnement des activités existantes; cet ordre local devant être fondé sur la négociation des conditions d'application des dispositions juridiques, mais aussi sur des demandes d'adaptation des procédures administratives et de police, des règles secondaires d'application'. Par ailleurs, on observe la multiplication d'instances qui affichent l'objectif de reconstruire la confiance entre acteurs à partir d'un débat et d'accords sur les règles et leur application, éventuellement en intégrant la vigilance des habitants mobilisés ou «l'expertise d'usage » des riverains.

Dans le cadre des conflits de proximité, les habitants font l'expérience que le droit ne s'applique pas seul, qu'il est nécessaire de le faire exister localement, travail pris en charge par certains acteurs. Pour acquérir un rôle dans une situation, le cadre juridique doit faire l'objet d'un travail de localisation, de construction sociale de sa validité locale, d'actualisation locale au sein de processus de régulation sociale. A ce niveau d'analyse, nos travaux montrent d'abord que pour les collectifs mobilisés le droit apparaît comme un élément externe qu'il faut rendre présent dans les situations locales. Les conflits analysés ici produisent donc d'une façon directe ou symbolique une actualisation locale du droit. Nous utilisons ici la notion d'actualisation au sens d'action de faire passer de l'état virtuel à l'état réel pour caractériser la façon dont des règles de droit, des procédures, peuvent être activées, importées dans une situation lorsque certains acteurs les font exister en les mobilisant symboliquement ou pratiquement. Il s'agit d'une définition proche de la notion de "localisation» ou (re)«localisation» chez Anthony Giddens, c'est-à-dire « d'enracinement (même partiel ou provisoire) dans un contexte spatio-temporel local » et dans des échanges de face à face de relations sociales ou de processus qui avaient été délocalisés, c'est-à-dire "détachés des contextes locaux» (Giddens, 1994 : 85).

30 Cette notion permet de distinguer, dans le cas des transactions autour de la construction de règles locales, les processus d'accomplissement situés du droit, de ceux qui constituent une production locale de droit. Des travaux sur des situations de conflits en France, ont en effet montré que les tentatives d'élaboration négociée de chartes locales qui se présentent comme une production juridique locale constituaient en fait des processus d'actualisation du droit (Melé, 2006). En effet, si les chartes étudiées n'introduisent que peu d'éléments supplémentaires par rapport à la législation, elles miment néanmoins la production juridique. Pour les associations - comme pour les pouvoirs publics - le moment de la négociation est plus important que celui de la signature de la charte. Censées assurer la puissance publique de la qualité de la localisation de l'ordre juridique, actualisé par la participation et une rédaction mobilisant les parties, elles constituent pour les associations un moment de reconnaissance de leur capacité à peser sur le cadre de régulation des activités. Un des effets de la rédaction de ces textes, même s'ils sont souvent non signés par l'ensemble des participants, semble être de diffuser des schémas d'interprétations partagés des situations. Ces éléments, nous ont conduits à préférer la notion d'ordre juridique localisé (issu de processus de «localisation » ou d'actualisation locale) à celle d'ordre juridique local (issu de processus de production locale), pour caractériser ce qui était en jeu dans les processus locaux de régulation analysés dans Les règles du jeu urbain (Bourdin, Lefeuvre, Melé, 2006).

31 Pour activer localement les opportunités offertes par le cadre juridique, les groupes mobilisés nécessitent des capacités d'action et des compétences qui sont inégalement 
réparties. Les collectifs qui impliquent des classes moyennes, des anciens employés de l'état ou des militants environnementalistes ou politiques semblent plus «armés » pour adopter une stratégie systématique d'évocation du droit ou de recours juridiques, c'est vrai dans la situation mexicaine comme française. De plus, nos entretiens soulignent aussi l'importance de la qualité des spécialistes du droit mobilisés et des limitations introduites par le coût financier des recours.

Or, pour que le droit soit mobilisable, il faut que le cadre juridique offre des prises aux opposants. Une partie de la capacité des collectifs locaux à mobiliser le droit est liée aux évolutions juridiques dans le domaine de l'urbanisme ou de l'environnement, des procédures de participation ou de concertation ou de transparence. A cet égard, les contextes nationaux offrent des niveaux de prise très différents, en termes de capacité à agir comme de protection éventuelle apportée par un jugement.

Le droit n'est donc pas une entité extérieure qui saisit des situations, les qualifie et détermine ainsi un règlement des usages et des pratiques. Non seulement les juges et les représentants des pouvoirs publics n'ont pas le monopole de l'interprétation, mais les situations de conflits instaurent des transactions autour du droit, de ses objectifs et de son application. De plus, les conditions de sa validité locale sont définies au sein d'interactions et de processus d'actualisation locale.

Ces réflexions pourraient être le point de départ de futures recherches qui se proposeraient d'explorer comment l'expérience du droit et de ces processus d'actualisation locale laissent une marque sur un espace ou une société locale et peuvent constituer une référence pour des actions futures. Existe-t-il, à partir de telles expériences, des apprentissages qui produisent des stratégies différentes, qui modifient les formes de mobilisation?

\section{Rôle des qualifications juridiques de l'espace}

En ce qui concernent les relations entre droit et processus de territorialisation liés aux situations de conflits, nos travaux ont aussi mis en évidence que pour les habitants mobilisés l'épreuve du conflit a aussi été un moment de confrontation à différentes formes de qualifications juridiques de l'espace (zonages), outils du droit et modes de spatialisation de l'action publique. En effet, la présence dans un grand nombre de contextes d'espaces protégés ou identifiés pour leurs qualités environnementales et patrimoniales constitue une ressource pour les habitants mobilisés (Melé, 2008). Dans leurs recherches d'éléments permettant d'identifier les qualités des espaces qu'ils cherchent à défendre, les groupes mobilisés rencontrent ces formes de territorialisation des valeurs patrimoniales et environnementales. Si ces dispositifs sont relativement peu connus des populations, les habitants mobilisés deviennent des spécialistes de l'usage des cartes et de l'utilisation de ces zonages pour décrire les qualités du territoire. Il s'agit pour eux souvent de mettre en avant des incohérences entre des projets présentés comme inconciliables pour un même espace; de rechercher les incompatibilités d'images ou réelles entre définitions et qualifications des usages de l'espace.

36 Alors que les stratégies esthétiques ou de valorisation de l'image des lieux peuvent être sujets de débats et de controverses sur les limites pertinentes ou sur la comptabilité avec le projet, les qualifications de l'espace ancrées en droit constituent en revanche les seuls éléments de territorialisation des valeurs de l'espace qui peuvent être considérés comme 
«non négociables $»^{10}$. Le juge administratif peut s'appuyer sur les espaces protégés, ou même sur l'identification de qualité environnementale sans protection comme dans le cas des $\mathrm{ZNIEFF}^{11}$, pour trouver une définition partagée de la valeur des espaces et parfois donner raison aux opposants en statuant sur une « erreur manifeste d'appréciation » des documents d'aménagement autorisant la mise en place d'un projet. Un effet du conflit est ici de placer le juge dans la situation de devoir rendre actif et de donner un contenu à des espaces protégés, ou à des qualifications, dont les impacts juridiques n'étaient pas directs.

Pour ancrer, pour donner un contenu à leur recherche de valeurs de l'espace affecté mobilisable les résidents tentent de se rattacher à des qualifications institutionnelles dont l'activation pourrait permettre de créer une incompatibilité avec le projet.

La mobilisation de l'espace comme élément affecté par un projet peut être perçue comme une stratégie pertinente pour ancrer des demandes de protection au sein de l'action publique environnementale. Dans plusieurs cas, les personnes interrogées construisent une critique des politiques environnementales. Celles-ci, focalisées sur la protection des espèces et des espaces méconnaitraient les dimensions sanitaires et la protection des personnes face aux risques. Certains regrettent de ne pas habiter plus près d'un parc ou d'un espace protégé ou même, dans un cas, de ne pas être eux-mêmes « des espèces protégées ou des vestiges archéologiques ou patrimoniaux » (Cirelli, 2013).

Lorsque des collectifs cherchent à mobiliser une qualification juridique existante pour renforcer leur position, on assiste à une stratégie d'élargissement des capacités d'action grâce aux acteurs publics et aux ressources juridiques liées à certaines formes d'espaces protégés, il s'agirait alors d'une stratégie d'empowerment par l'espace. Lorsque des habitants se mobilisent pour la mise en place d'une qualification juridique sur un sousensemble spatial, on pourrait considérer qu'il s'agit de la volonté de doter certains sousespaces de qualités juridiques pouvant venir renforcer leurs revendications et leurs positions.

\section{Conclusion}

40 Le droit joue donc un rôle important dans les conflits, non seulement comme ressource mais aussi comme cadre cognitif. Certes, il ouvre un canal pour la judiciarisation des différends; une grande partie des conflits peuvent avoir une dimension de contentieux. Mais même en dehors de toute plainte ou recours, le droit contribue à construire des attentes, à déterminer des positions et constitue une ressource argumentative, dans le cadre de processus locaux d'actualisation dans lesquels des dispositifs institutionnalisés tiennent une place différente.

41 Or, il existe un autre type de processus juridique qui transcende le conflit mais qui surgit du conflit, et qui en principe, peut affecter des conflits futurs qui n'a pas été traité ici. Il s'agit des changements dans la législation ou dans les modalités de sa mise en œuvre. Nous évoquons ici le lien entre conflit et juridicisation. Des processus législatifs ou réformes administratives sont, explicitement ou implicitement, marqués par l'idée que certains conflits ne sont pas traités correctement ou que des activités doivent être plus strictement encadrées pour éviter la conflictualité liée aux externalités négatives. Certaines réformes ou nouvelles lois peuvent avoir comme objectif explicite ou implicite d'éviter que des situations ne se reproduisent, en particulier à cause de leur caractère conflictuel. Alors que dans la littérature scientifique, les explications du changement 
institutionnel tendent à coïncider sur l'idée que les transformations des règles juridiques sont le produit d'un dialogue entre acteurs dégagés de leurs positions et intérêts - depuis la "gouvernance" pour la science politique jusqu'aux droits humains de troisième génération pour la philosophie du droit - une des leçons de nos travaux est de rappeler que les changements institutionnels ne sont pas seulement le résultat de la seule volonté d'acteurs rationnels qui ont participé à leurs productions.

Nos recherches n'avaient pas pour objectif d'étudier ces processus législatifs. Néanmoins pour poursuivre la réflexion sur la productivité juridique des conflits, et en particulier pour intégrer les effets de long terme, il semble nécessaire d'introduire une réflexion pour saisir les liens entre conflictualité et production juridique. Cela nécessiterait de construire des hypothèses sur la façon dont une situation de conflit peut dépasser le niveau local et se convertir en une référence justifiant une production législative ou l'adaptation de nouvelles procédures administratives.

\section{BIBLIOGRAPHIE}

Arnaud A. J., 1993, Dictionnaire encyclopédique et théorique du droit, Paris, LGDJ, $2^{\mathrm{e}}$ édition.

Azuela A., 2006, Visionarios y pragmáticos: una aproximación sociológica al derecho ambiental, México, Mexique, Universidad Nacional Autónoma de México, distrib. Fontamara.

Bayón J. C., 2000, « Derrotabilidad, indeterminación del derecho y positivismo jurídico », Isonomîa , no 13 , p. 87-117.

Bobbio L., Melé P., Ugalde V., 2015, Décider en situation de crise : gestion des déchets, conflits et concertations (France, Italie, Mexique), Manuscrit issu du rapport pour le programme Concertation, décision, environnement, Ministère de l'écologie, ADEME, UMR CITERES en cours de publication. Bouba-Olga O., Carrincazeaux C., Coris M., 2008, « Avant propos, La proximité 15 ans déjà », Revue d'économie régionale et urbaine, volume 3, p. 279-287.

Bourdin A., Lefeuvre M.-P., Melé P., 2006, Les règles du jeu urbain, entre droit et confiance, Paris, Descartes et cie.

Cirelli C., 2013, « «Elle était petite, elle ne se voyait pas » : mobilisation et conflit autour d'une décharge en Isère », in Melé P. (dir.), 2013, Conflits de proximité et dynamiques urbaines, Rennes, Presses universitaires de Rennes, p. 95-134.

Comanducci P., 1998, «Principios jurídicos e indeterminación del derecho », Doxa, volume 2, n 21, p. 89-104.

Giddens A., 1994, Les conséquences de la modernité, Paris, Puf, 192 p.

Hart, H.L.A. 2006, Le concept du droit, Bruxelles, Publications des Facultés Universitaires SaintLouis.

Kelsen H., 1962, Théorie pure du droit, Paris, Dalloz, Paris.

Kress K. J., 1989, « Legal Indeterminacy », California Law Review, vol. 77, n², p. 283-337. 
Lascoumes P. et Serverin E., 1986, « Théories et pratiques de l'effectivité du droit », Droit et Société, $\mathrm{n}^{\circ} 2$, p. 127-150.

Lascoumes P., 1990, « Normes juridiques et mise en œuvre des politiques publiques », L'année Sociologique, 1990, p. 40-71.

Latour B., 2002, La fabrique du droit, une ethnographie du Conseil d'État, Paris, La découverte.

Melé P. (éd.), 2013, Conflits de proximité et dynamiques urbaines, Rennes, Presses universitaires de Rennes.

Melé P., 2006, «Lutter contre les bruits de la ville, mobilisation du droit et production d'ordres locaux ", in, Boudin A, Lefeuvre M-P., Melé P., Les règles du jeu urbain entre droit et confiance, Paris, Descartes et cie, p. 207-242.

Melé P., 2008, « Territoires d'action et qualifications de l'espace », in, Melé P., Larrue C., Territoires d'action, Paris, L'Harmattan, p. 15-45.

Melé P., 2011, Transactions territoriales : patrimoine, environnement et actions collectives au Mexique, Tours, PUFR, $213 \mathrm{p}$.

Melé P., Larrue C., 2008, Territoires d'action, Paris, L’Harmattan.

Redondo M. C., 1997, « Teorías del Derecho e Indeterminación Normativa », Doxa, n²0, p. 177-196.

Rottlethuner H. 1989, « A Purified Sociology of Law: Niklas Luhmann on the Autonomy of the Legal System », Law \& Society Review, Vol 23, Núm 5.

Rocher L., 2006, Gouverner les déchets: gestion territoriale des déchets ménagers et participation publique, thèse de doctorat d'aménagement, CITERES, Université François Rabelais, 445 p.

Teubner, G., 1989, « Et Dieu rit. Indétermination, autoréference et paradoxe en droit », Archives de philosophie du droit, Vol 34, 1989, pp. 269-294.

Teubner G., 1991, « The end of Regulatory Law: Chronicle of a Death Foretold » Social \& Legal Studies, Vol. 1 Num 4. p. 451-475.

Torre A., 2009, « Retour sur la proximité géographique », Géographie Économie Société, 2009, vol. 11, p. 63-75.

Torre A., Melot R., Bossuet L. et all. 2010, « Comment évaluer et mesurer la conflictualité liée aux usages de l'espace? « Vertigo - Revue. électronique en sciences de l'environnement, Volume 10 Numéro 1, < http://dx.doi.org/10.4000/vertigo.9590 >

\section{ANNEXES}

Annexe : Travaux de terrain

Les considérations développées dans cet article sont le résultat d'échanges entre les auteurs et d'autres chercheurs dans le cadre de deux projets collectifs internationaux basés sur l'analyse croisée d'études de cas. Par ailleurs, deux journées d'études internationales ont été réalisées en avril 2013 par l'UMR CITERES avec l'aide du STUDIUM. On trouvera sur le site de l'UMR CITERES une présentation détaillée des institutions et des chercheurs qui ont participé à ces projets. 
1 - Le projet CONFURB : Conflits de proximité et dynamiques urbaines (France, Mexique, Canada), P. Melé (coord). pour l'ANR SHS « Conflits, guerre(s), violence» de l'ANR-SHS » (2007-2011), dont les résultats ont été publiés dans l'ouvrage Conflits de proximité et dynamiques urbaines (Melé 2013) avait choisi de diversifier les types de conflits. Les études de cas en France ont concerné des situations dans lesquelles des groupes de résidents se mobilisaient pour :

a) Lutter contre risques, pollutions, nuisances

- La mobilisation des riverains contre l'extension de la décharge de Saint-Alban à Vienne, Isère (Claudia Cirelli).

- Les tensions dues à la proximité d'une zone industrielle avec le secteur résidentiel du Grand Village (La-Ville-aux-Dames) dans l'agglomération de Tours (José Serrano).

b) Lutter contre des projets publics d'infrastructures

- Les oppositions à la rocade sud d'Angers (Laurence Rocher).

- Les mobilisations contre les effets de la pénétration dans l'agglomération de Bordeaux des voies ferrées à grande vitesse (Laurence Rocher).

c) Rester

- Une mobilisation fragile contre un projet de rénovation urbaine (la Verrerie à Amboise) (Hélène Bertheleu).

À Montréal, deux situations ont été choisies en fonction des contextes urbains (quartiers péricentraux, banlieue) mobilisant des formes différentes d'institutionnalisation des débats et portant sur des objets distincts.

- L'espace public en contexte socio-ethnique contrasté : le cas de la Place G. Caboto à Saint-Léonard (Annick Germain).

- Le cas de mobilisation d'artistes et artisans pour continuer à occuper l'ancienne usine Grover dans le quartier Sainte-Marie (Geneviève Cloutier).

À Mexico, les chercheurs membres du projet ont choisi de concentrer leurs efforts sur un type de contexte urbain celui des anciens villages, qui permet de saisir des controverses liées à la dynamique de l'urbanisation.

- La mobilisation des résidents et des membres des communautés agraires de San Salvador Atenco et des villages proches contre l'expropriation de terres pour la réalisation du nouvel aéroport de la ville de Mexico (Antonio Azuela).

- L'analyse des conflits et des tensions entre les anciens habitants et une enclave résidentielle de haut niveau d'inspiration environnementale (Tlalpuente) qui occupe une aire boisée qui appartenait au village de San Andres Totoltepec dans l'Ajusco (Angela Giglia).

2 - Le projet DeSCRI, Décider en situation de crise : gestion des déchets, conflits et concertations (France, Italie, Mexique) pour le programme CDE, Concertation, décision, environnement, Ministère de l'écologie, de l'énergie et du développement durable, ADEME (2008-2012) s'est focalisé sur des situations d'oppositions ou de controverses liées à des infrastructures de traitement des déchets domaine caractérisé à la fois par l'ampleur des blocages et les évolutions rapides des législations dans tous les contextes nationaux analysés. Notons aussi que le travail sur cet article a été rendu possible par des 
missions Mexique-France et France-Mexique financées par le programme ECOS-Nord. Les situations étudiées par le projet DeSCRI sont toutes marquées par le blocage de projets de réalisations ou d'extension de décharge ou d'incinération.

1 - France :

Décision, planification et conflits en Indre-et-Loire. Étude de l'échec d'une tentative de localisation d'un incinérateur pour l'agglomération de Tours (Laurence Rocher).

Conflits et décision dans le département de l'Isère. L'analyse de deux conflits liées à des installations de stockage de déchets non dangereux : ménagers à Vienne, inertes à Izeaux (Claudia Cirelli).

2 - Italie :

Un deuxième incinérateur pour la Province de Turin (Matteo Puttilli, Nadia Tecco).

L'incinérateur de l'agglomération de Gênes (Gianfranco Pomatto).

L’opposition à la construction de l'incinérateur de Palerme (Fabrizio Maccaglia).

3 - Mexique :

La crise de la gestion des déchets à Cuernavaca (Ana Lorena Gurza).

Le projet de centre intégral de recyclage et d'énergie (CIRE) de Tlahuac dans la ville de Mexico (Jade Latargère).

L'implantation d'un centre de stockage des déchets dangereux à Zimapán, Hidalgo

(Vicente Ugalde).

\section{NOTES}

1. Ce vocabulaire n'implique donc pas une adhésion des auteurs à l'école de la proximité en économie (Bouba-Olga, Carrincazeaux et Coris, 2008, Torre 2009), même si certaines questions peuvent être communes avec des chercheurs travaillant dans cette optique (Torre, Melot, Bossuet et all, 2010).

2. Cf. Annexe : Travaux de terrain

3. La théorie des systèmes sociaux propose le questionnement le plus fort aux attentes et à la croyance dans la fonction régulatrice du droit. Cf. la polémique entre G. Teubner (1991) et H. Rottlethuner (1989).

4. Cf. le cas de l'implantation d'une décharge de déchets dangereux à Guadalcázar (Azuela, 2006)

5. Cf. Comanducci, 1998 ; Redondo, 1997; Bayón, 2000; Kress, 1989 ; Teubner, 1989.

6. Concept utilisé en linguistique et sociologie du droit pour caractériser le fait que le sens des mots n'est pas fixe mais contextuel et donc sujet à interprétation.

7. En particuliers, en France, en ce qui concerne les modalités de définition pratique de la notion de « déchet ultime " (Cirelli, 2013 ; Rocher, 2006)

8. En instaurant une capacité à agir pour des individus ou pour des collectifs qui ne sont pas directement touchés.

9. «principes pratiques développés par les agents publics pour assurer la mobilisation et l'adaptation des règles étatiques aux faits sociaux qu'il leur appartient de gérer » : instructions, circulaires, procédures qui sont présentées aux usagers comme la mise en œuvre du droit » (Lascoumes, Pierre, 1990 : 56). 
10. Au sens ici de la robustesse des limites ancrées en droit, il faut bien sûr nuancer ce propos en rappelant que leurs délimitations, même lorsqu'elles se présentent comme ancrées dans les valeurs environnementales ou les risques sont l'objet de négociations, d'ajustements, comme l'ont montré les différents textes réunis sur cette question dans Territoires d'action (Melé, Larrue 2008).

11. Les Zones naturelles d'intérêt écologique faunistique et floristique ont été délimitées suite à un processus lancé en 1982 dans l'objectif d'identifier sur l'ensemble du territoire français les secteurs présentant de fortes capacités biologiques et un bon état de conservation.

\section{RÉSUMÉS}

Le droit joue un rôle important dans les conflits de proximité, non seulement comme ressource mais aussi comme cadre cognitif. Certes, il ouvre un canal pour la judiciarisation des différends ; une grande partie des conflits peuvent avoir une dimension de contentieux. Mais même en dehors de toute plainte ou recours, le droit contribue à construire des attentes, à déterminer des positions et constitue une ressource argumentative, dans le cadre de processus locaux d'actualisation dans lesquels des dispositifs institutionnalisés tiennent une place différente. Cet article propose d'intégrer aux débats sur les dimensions juridiques des relations à l'espace et sur les dimensions spatiales et territoriales du droit, des hypothèses d'interprétation sur la place du droit dans les situations de conflits de proximité issu de plusieurs projets de recherche internationaux.

The law plays an important role in land-use conflicts that mobilize people against projects close to their neighborhoods ("proximity conflicts"), not only as a resource but also as a cognitive framework. It is true that the law creates opportunities for bringing controversies to the judiciary, as a growing proportion of those controversies end up in litigation. But even outside the courtroom the law provides argumentative resources to social actors, contributes to organize their expectations, and defines their positions. All this can be seen in the different ways legal devices are brought in by social actors. This article elaborates on these issues from the results of several international research projects on proximity conflicts and suggests hypotheses on how to interpret the place of law in the social productivity of those conflicts.

\section{INDEX}

Keywords : proximity conflicts, land use conflicts, law, legal classification of space, litigation

Mots-clés : conflits, proximité, ordre juridique, qualifications juridiques de l'espace, contentieux

\section{AUTEURS}

\section{ANTONIO AZUELA}

Antonio Azuela est juriste et sociologue, chercheur de l'Institut de recherches sociales de l'Université national autonome de Mexico. Il travaille actuellement sur la formation du régime 
du patrimoine naturel au Mexique et sur le rôle des juges dans les conflits urbains en Amérique Latine.

\section{PATRICE MELÉ}

Patrice Melé est géographe, professeur des universités, UMR CITERES, CNRS-Université de Tours. Il travaille sur les mutations des rapports au territoire sous l'effet de la diffusion du patrimoine et de l'environnement comme valeurs et cadres pour l'action publique.

\section{VICENTE UGALDE}

Vicente Ugalde, juriste et spécialiste des études urbaines, professeur-chercheur au CEDUA / El Colegio de Mexico. Ses intérêts de recherche portent sur l'analyse de la mise œuvre des politiques environnementales et la prise de décisions dans le contrôle de la réglementation environnementale. 